\title{
WSN-UAV Monitoring System with Collaborative Beamforming and ADS-B based Multilateration
}

\author{
Yogesh Nijsure $^{\dagger}$, Mohammed F. A. Ahmed, Georges Kaddoum, Ghyslain Gagnon, Francois Gagnon \\ $\dagger$ Corresponding author, e-mail: y.nijsure.2014@ieee.org
}

\begin{abstract}
This paper presents wireless sensor networkunmanned aerial vehicle (WSN-UAV) system for military remote monitoring and surveillance. Large scale WSN is deployed in a battlefield or wide hostile region to collect information of interest and send it to a UAV. Collaborative beamforming $(\mathrm{CB})$ is used to achieve the ground-to-air transmissions. An automatic dependent surveillance-broadcast (ADS-B) based multilateration is used to obtain the UAV location and tracking information. It is found that a minimum distance between the UAV and the WSN is required for proper operation of the $\mathrm{CB}$ due to the precision of the multilateration and the movement of the UAV.
\end{abstract}

Index Terms-Monitoring and surveillance, Geolocation, ADSB based multilateration, Large scale wireless sensor networks, Collaborative beamforming.

\section{INTRODUCTION}

Wireless sensor networks (WSNs) is an attractive tool for monitoring and surveillance applications due to its flexible deployment and ability of unattended operation [1], [2]. Sensor nodes are deployed in an ad-hoc fashion over large and remote area to collect information from the surrounding neighbourhood and send it to a fusion center (FC) for processing and decision making. In order to have reasonable cost, sensor nodes are fabricated with off-the-shelf microcontrollers and, consequently, have limited processing capability. Moreover, energy is typically supplied in sensor nodes from batteries or energy harvesting devices [3].

Sensor nodes are deployed close to the event or phenomena under surveillance which is typically far from the FC. Data gathering can be implemented using unmanned aerial vehicle (UAV) as a FC. The UAV flies over the sensing field while sensor nodes, which are deployed on the ground, transmit the collected data directly to the UAV. UAVs have the advantage to explore vast geographical region.

In this paper, we focus on military tactical monitoring and surveillance to remotely monitor the battlefield or wide hostile regions [4]. In such scenarios, a UAV is utilized as the FC which is expected to fly at high altitudes. The distances between the sensor nodes and the UAV can be larger than the transmission range of individual sensor node. In this case, collaborative beamforming (CB) [5], [6] is utilized for ground-to-air transmission in the proposed system to increase the transmission range and achieve directional gain. In order to implement $\mathrm{CB}$, the location and tracking information of the UAV should be available at the sensor nodes. Typically, global positioning system (GPS) can be used to obtain the UAV location information and broadcast it to the sensor nodes. However, we are interested in military and tactical applications where there is a threat of GPS jamming, spoofing and message infringement type attacks. In this case, other navigation and guidance subsystems can be used to obtain this information [4]. Recently, automatic dependent surveillancebroadcast (ADS-B) has been introduced to support avionics navigation. The ADS-B signal can carry location information relative to local point in the flying path. Additionally, the ADS-B signal is captured at ground receivers and used for

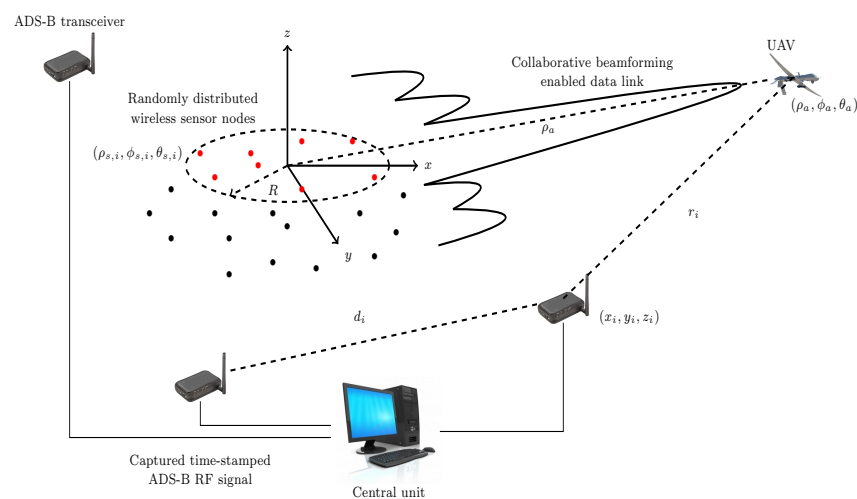

Fig. 1. The geometric model of the system.

passive localization using time-difference-of-arrival (TDOA) [7], [8]. Such a robust framework for uninhibited tracking of the UAV through ADS-B based multilateration does not rely upon the message content of the ADS-B signal and uses the captured ADS-B signal to achieve 3D geolocation. The effect of the error bounds of ADS-B based multilateration and the UAV movement on the CB performance is considered.

The rest of the paper is organized as follows. In Section II, we provide a general overview of the proposed WSN-UAV remote surveillance system architecture. In Section III, we present the ADS-B based multilateration for UAV tracking in real-time. In Section III-B, CB for the uplink transmission is presented. Simulation results are described in detail in Section IV, which illustrate the efficiency of the $\mathrm{CB}$ enabled by the ADS-B based multilateration. Finally, concluding remarks are provided in Section V.

\section{SYSTEM ARCHITECTURE}

A large scale WSN is deployed by, for example, dropping the sensor nodes from an aircraft over the area of interest as shown in Fig. 1. Sensor nodes know their local coordinates from a UAV self-localization scheme [9]. The location information is used to organize the nodes into clusters where each cluster consists of $N$ sensor nodes located close to each other inside a circle of radius $R$.

Consider the local spherical coordinates $(\rho, \phi, \theta)$ for each cluster where the origin point is the center of cluster, the angle $\theta$ denotes the elevation direction, $\phi$ represents the azimuth direction, and $\rho$ is the distance from the origin to a given point. Let $\left(x_{a}, y_{a}, z_{a}\right)$ be the current position of the UAV which corresponds to $\left(\rho_{a}, \phi_{a}, \theta_{a}\right)$ in the spherical coordinates. ADS$B$ receivers are located away from the battlefield and separated with large distances, typically over $18.5 \mathrm{~km}$ [10]. Let the $i$ th receiver to be located at $\left(\rho_{i}, \phi_{i}, \theta_{i}\right)$. Sensor nodes are deployed on the ground, i. e., co-located in the $x y$-plane, with rectangular coordinates $\left(x_{s, i}, y_{s, i}, 0\right), i=1, \ldots, N$ and the corresponding spherical coordinates are $\left(\rho_{s, i}, \phi_{s, i}, \pi / 2\right)$. The distances between the sensor nodes in one cluster are small 
enough to neglect the power consumed for communication within the cluster [11]. We also assume that the sensor nodes are frequency synchronized and frequency drift effects are negligible [12]. All sensor nodes within a cluster have access to the data/measurements to be transmitted to the UAV. This information sharing is simply performed by broadcasting the data from one sensor node to all other nodes in its cluster [5], [13], [14]. There is a line-of-sight (LOS) between the sensor nodes and the UAV and the environment does not have any obstacles. Therefore, the effects of the reflection, scattering, and shadowing on the signal are very small and the channel fading follows Rician distribution with high $K$-factor [15]. Moreover, the transmitted signal decays with distance due to the propagation in a LOS path according to the free-space path loss (FSPL).

The UAV is equipped with ADS-B transponder that transmits at $1090 \mathrm{MHz}$ every second without the need of any ground based interrogative signals. These signals are synchronously captured by the ADS-B receivers which are synchronized by rubidium or cesium based clocks and can provide a timing synchronization accuracy of $20 \mathrm{~ns}$ [16]. The proposed system is based on TDOA localization and does not rely upon the demodulation or decoding of the ADS-B message transmitted by the UAV, it only requires the raw RF ADS-B signal. The RF ADS-B signal is captured at the receiver units and time-stamped to allow for cross-correlation in order to evaluate the corresponding TDOA profiles. This TDOA profile is computed at the central unit (CU) located at the command center and a $3 \mathrm{D}$ geolocation estimate is generated for the current UAV position. This estimate is passed on to the tracking unit based on extended Kalman filter (EKF) to enable the real-time trajectory tracking for the UAV. The geolocation estimate and the tracking estimate of the UAV is transmitted back to the UAV through the ADS-B uplink at $1030 \mathrm{MHz}$. The UAV then broadcasts its current geolocation and tracking estimate to the sensor nodes using high power direct transmission. The UAV flies over the WSN to collect the surveillance data with time-slotted transmissions. Based on the geolocation information, the clusters of sensor nodes identify their distance to the UAV. Only one cluster is allowed to transmit at each time slot depending on its distance from the UAV. The clusters keep updated with the geolocation and real-time tracking estimate of the UAV and based on that estimate, the next cluster of sensor nodes activates for $\mathrm{CB}$ transmission. The CB thus benefits from the real-time tracking of the geolocation estimate for the UAV.

\section{THE PROPOSED WSN-UAV SYSTEM}

\section{A. ADS-B based multilateration for real-time UAV tracking}

A time vector is appended to the captured ADS-B signal at all the ADS-B receivers, and this time-stamped signal is sent to the $\mathrm{CU}$ for TDOA processing. At the CU, the time-stamps are aligned to cross-correlate the signals and compute the time-difference profiles to enable TDOA computation. Let $r_{i}$ be the distance between the UAV and the $i$ th ADS-B receiver, then we can write

$$
r_{i}=\sqrt{\left(x_{a}-x_{i}\right)^{2}+\left(y_{a}-y_{i}\right)^{2}+\left(z_{a}-z_{i}\right)^{2}} .
$$

The TDOA between sensors $i$ and $j$ is then defined by $\Delta \tau_{i, j}$

$$
\Delta \tau_{i, j}=\tau_{i}-\tau_{j}=\frac{1}{c}\left(r_{i}-r_{j}\right)
$$

where $c$ is the speed of light. TDOAs are assumed to be obtained with respect to first ADS-B receiver, thus

$$
\Delta \tau_{i, 1}=\frac{r_{i}-r_{1}}{c}+\eta_{\Delta_{\tau_{i, 1}}}, \quad i=2, \ldots, k,
$$

where $\eta_{\Delta_{\tau_{i, j}}}$ is the noise associated with $\Delta \tau_{i, 1}$. In the vector form

$$
\mathbf{m}=\mathbf{g}(\mathbf{X})+\eta,
$$

where $\mathbf{X}=\left[x_{a}, y_{a}, z_{a}\right]^{T}$ is the vector of unknown variables, $\mathbf{m}=\left[\Delta \tau_{2,1}, \Delta \tau_{3,1}, \cdots, \Delta \tau_{k, 1}\right]^{T}$, $\mathbf{g}(\mathbf{X})=\left[\left(r_{2}-r_{1}\right) / c,\left(r_{3}-r_{1}\right) / c, \ldots,\left(r_{k}-r_{1}\right) / c\right]^{T}$, and $\eta=\left[\eta_{\Delta_{\tau_{1,1}}}, \eta_{\Delta_{\tau_{2,1}}}, \ldots, \eta_{\Delta_{\tau_{k, 1}}}\right]^{T}$.

Consequently, the TDOA estimation problem can be expressed as follows,

$$
\hat{\mathbf{X}}=\arg \min _{\mathbf{X}}\|(\mathbf{m}-\mathbf{g})\|,
$$

where $\hat{\mathbf{X}}$ is the TDOA estimate of the UAV location. Equation (5) can be solved using any least squares minimization algorithm such as the Levenberg-Marquardt algorithm [17]. Additionally, we adopt the EKF tracking algorithm of [18]. For simulation purposes, we model the state of the EKF by incorporating the position and velocity of the moving UAV. The distance from the ADS-B receiver units can be expressed as

$$
d_{i, t}=\sqrt{\left(x_{a, t}-x_{i}\right)^{2}+\left(y_{a, t}-y_{i}\right)^{2}+\left(z_{a, t}-z_{i}\right)^{2}} .
$$

One way of modelling the motion is to set up a linear system composed of the kinematic equations for each dimension of the tracked movement.

In the following, the UAV's $3 \mathrm{D}$ motion is represented by its position and velocity at time step $t=T$ which corresponds to the current state $\mathbf{X}_{T}$ in the EKF formulation, the state vector can be expressed as

$$
\mathbf{X}_{T}=\left(\begin{array}{c}
x_{a, T} \\
y_{a, T} \\
z_{a, T} \\
\dot{x}_{a, T} \\
\dot{y}_{a, T} \\
\dot{z}_{a, T}
\end{array}\right)=\mathbf{C} \cdot\left(\begin{array}{c}
x_{a, T-1} \\
y_{a, T-1} \\
z_{a, T-1} \\
\dot{x}_{a, T-1} \\
\dot{y}_{a, T-1} \\
\dot{z}_{a, T-1}
\end{array}\right)+\mathbf{E}
$$

where $\dot{x}_{a, T}, \dot{y}_{a, T}$ and $\dot{z}_{a, T}$ are the velocities in the $x, y$ and $z$ directions, $\mathbf{C}$ is the state transition matrix for the EKF, and $\mathbf{E}$ is the process noise vector that accounts for any un-modelled factors in the system. At time step $t=T$, let $\tilde{d}_{i, T}(i=$ $1, . ., K)$ be the distance measurement errors. The measured distances are given by

$$
\begin{aligned}
d_{i, T} & =\sqrt{\left(x_{a, T}-x_{i}\right)^{2}+\left(y_{a, T}-y_{i}\right)^{2}+\left(z_{a, T}-z_{i}\right)^{2}} \\
& +\tilde{d}_{i, T}, \quad i=1,2, \ldots, K .
\end{aligned}
$$

The above equation can also be written in vector form as

$$
\left(\begin{array}{c}
d_{1, T} \\
d_{2, T} \\
\vdots \\
d_{K, T}
\end{array}\right)=\mathbf{S X}_{T}+\left(\begin{array}{c}
\tilde{d}_{1, T} \\
\tilde{d}_{2, T} \\
\vdots \\
\tilde{d}_{K, T}
\end{array}\right)
$$

where $\mathbf{S}$ is the measurement matrix that relates the current state to the output. Since the outputs of (8) are non-linear, 
the Jacobian should be used to linearize and facilitate the computation of (7) [18]. Hence,

$$
\mathbf{S}=\left(\begin{array}{cccccc}
\frac{\partial d_{1, T}}{\partial x} & \frac{\partial d_{1, T}}{\partial y} & \frac{\partial d_{1, T}}{\partial z} & 0 & 0 & 0 \\
\frac{\partial d_{2, T}}{\partial x} & \frac{\partial d_{2, T}}{\partial y} & \frac{\partial d_{2, T}}{\partial z} & 0 & 0 & 0 \\
\vdots & \vdots & \vdots & \vdots & & \\
\frac{\partial d_{K, T}}{\partial x} & \frac{\partial d_{K, T}}{\partial y} & \frac{\partial d_{K, T}}{\partial z} & 0 & 0 & 0
\end{array}\right)
$$

The following procedure can then be applied iteratively to track the UAV. In each iteration, five steps are performed as listed below [18].

1) Project the state ahead: $\mathbf{X}_{T}=\mathbf{C} \cdot \mathbf{X}_{T-1}$;

2) Project the error covariance ahead: $\mathbf{W}_{T}=\mathbf{C} \cdot \mathbf{W}_{T-1}$. $\mathbf{C}^{T}+\Omega$

3) Evaluate the Kalman gain: $\mathbf{K}_{T}=\mathbf{W}_{T} \cdot \mathbf{S}^{T}$. $\left(\mathbf{S} \cdot \mathbf{W}_{T} \cdot \mathbf{S}^{T}+\mathbf{R}\right)^{-1}$

4) Update estimation with measurements: $\mathbf{X}_{T}=\mathbf{X}_{T}+\mathbf{K}_{T}$. $\left(\mathbf{D}_{T}-\mathbf{S} \cdot \mathbf{X}_{T}\right)$

5) Update the error covariance: $\mathbf{W}_{T}=\left(\mathbf{I}-\mathbf{K}_{T} \cdot \mathbf{S}\right) \cdot \mathbf{W}_{T}$.

In Step 1 , the current state $\mathbf{X}_{T-1}$ is used to estimate the location at the next time instant. In Step 2, the error covariance matrix $\mathbf{W}_{T}$ in the next time step is projected using the state space model $\mathbf{C}$ and the process noise covariance matrix $\boldsymbol{\Omega}$. In Step 3, the Kalman gain $\mathbf{K}_{T}$ is computed, where $\mathbf{R}$ is a diagonal matrix representing the independent distance measurement noises at different ADS-B receivers. The Kalman gain is used in Step 4, when the distance measurements $\mathbf{D}_{T}=\left[d_{1, T}, d_{2, T}, \cdots, d_{K, T}\right]^{T}$ from the ADS-B receivers to the UAV are employed to update the state, $\mathbf{X}_{T}$. In Step 5 , the error covariance matrix $\mathbf{W}_{T}$ is updated. The current position $\left(x_{a, T}, y_{a, T}, z_{a, T}\right)$ is readily available from the state $\mathbf{X}_{T}$. Finally, the UAV receives the location information from the $\mathrm{CU}$ and broadcasts it to the sensor nodes. In the next section, we give details about $\mathrm{CB}$ transmission.

\section{B. $C B$ for the uplink transmission}

To implement CB transmission, sensor nodes use the same codebook with zero mean, unit power, and independent symbols, i.e., $\mathrm{E}\left\{s_{n}\right\}=0,\left|s_{n}\right|^{2}=1$, and $\mathrm{E}\left\{s_{n} s_{m}\right\}=0$ for $n \neq m$ where $s_{n}$ stands for the $n$th symbol from the codebook and $\mathrm{E}\{\cdot\}$ denotes expectation. Each collaborative node transmits the signal

$$
m_{i}=s \sqrt{P_{i}} e^{j \psi_{i}}, \quad i=1,2, \ldots, N
$$

where $s$ is the transmitted symbol, $P_{i}$ is the transmit power of the $i$ th sensor node, $\psi_{i}$ is the initial phase of the $i$ th sensor node's carrier to form the mainlobe of the beampattern towards the direction of the UAV.

The Euclidean distance between the $i$ th sensor node and a point on the sphere at which the UAV coordinates lay, i.e. point $\left(\rho_{a}, \phi, \theta\right)$, is

$$
\begin{aligned}
\delta_{r}(\phi, \theta) & \triangleq \sqrt{\rho_{a}^{2}+\rho_{s, i}^{2}-2 \rho_{s, i} \rho_{a} \sin (\theta) \cos \left(\phi-\phi_{s, i}\right)} \\
& \approx \rho_{a}-\rho_{s, i} \sin (\theta) \cos \left(\phi-\phi_{s, i}\right)
\end{aligned}
$$

where the approximation is valid for the far-field region with $\rho_{a} \gg \rho_{s, i}$. The corresponding phase delay is

$$
\varphi_{i}(\phi, \theta)=\frac{2 \pi}{\lambda} \delta_{i}(\phi, \theta),
$$

where $\lambda$ is the wavelength. Thus, using the knowledge of the sensor node location and the direction of the UAV, the initial phase of each sensor node carrier must be set as (see the closed-loop scenario in [5])

$$
\psi_{i}=-\frac{2 \pi}{\lambda} \delta_{i}\left(\phi_{a}, \theta_{a}\right)
$$

in order to achieve coherent combining at the UAV. Then, the received signal at direction $(\phi, \theta)$ from all sensor nodes in the cluster can be written as

$$
g(\phi, \theta)=s \sum_{i=1}^{N} \sqrt{P_{i}} e^{j \psi_{i}} h_{i} e^{j \varphi_{i}(\phi, \theta)}+n
$$

where $h_{i}$ is the channel fading between the $i$ th sensor node and the UAV and $n \sim \mathcal{C N}\left(0, \sigma_{n}^{2}\right)$ is the AWGN at the direction $(\phi, \theta)$ with variance $\sigma_{n}^{2}$. The received noise power $\sigma_{n}^{2}$ at UAV can be measured in the absence of data transmission and, therefore, is assumed to be known. The far-field beampattern corresponding to the sensor nodes in the cluster can be found as

$$
\mathbb{P}(\phi, \theta) \triangleq|g(\phi, \theta)|^{2}
$$

where $|\cdot|^{2}$ denotes the magnitude of a complex number.

At the UAV direction, the received signal is expressed as

$$
g\left(\phi_{a}, \theta_{a}\right)=s \sum_{i=1}^{N} \sqrt{P_{i}} h_{i}+n=s \mathbf{w}^{T} \mathbf{h}+n .
$$

where $\mathbf{w}=\left[\sqrt{P_{1}}, \sqrt{P_{2}}, \ldots, \sqrt{P_{N}}\right]^{T}$ is the weights vector and $\mathbf{h}=\left[h_{1}, h_{2}, \ldots, h_{N}\right]^{T}$ is the channel vector, and $(\cdot)^{T}$ denotes the transpose of a vector. The corresponding SNR $\gamma$ is given by

$$
\gamma=\frac{\left|\mathbf{h}^{T} \mathbf{w}\right|^{2}}{\sigma_{n}^{2}} .
$$

Without loss of generality, equal power allocation is used and the channel fading $h_{i}$ is normalized such that $\mathrm{E}\left\{h_{i}^{2}\right\}=1$. In this case, the propagation loss are due to the FSPL can be expressed as

$$
\mathrm{FSPL}=\left(\frac{4 \pi \rho_{a}}{\lambda}\right)^{2}
$$

and the effective path loss is the difference between the array gain and the FSPL (in $\mathrm{dB}$ ) is given by

$$
\mathrm{PL}=10 \log _{10}\left(\frac{4 \pi \rho_{a}}{\lambda}\right)^{2}-10 \log _{10}\left(N^{2}\right) .
$$

From the later expression, we can see that using more sensor nodes increases the array gain and reduces the effective path loss. However, increasing the cluster size $N$ for fixed sensor node density requires larger cluster radius $R$ which is practically limited by the transmission range of individual sensor node to allow data exchange within the cluster.

Equation (19) suggests that the closest cluster to the UAV, i.e., smallest $\rho_{a}$, should be allowed to transmit to reduce the effective path loss. However, the UAV does not have non-zero dimensions and we have to guarantee that the beampattern has an acceptable level at the whole UAV body. While the beampattern value at the UAV direction $\left(\phi_{a}, \theta_{a}\right)$ is totally deterministic, see (17), the CB beampattern in (15) at any direction $\left(\phi \neq \phi_{a}, \theta \neq \theta_{a}\right)$ is defined by the sensor node locations and it equals to the summation of the out-of-phase signals which is random. Fortunately, we are interested in the beampattern level at only the directions close to the UAV direction. The useful region of the beampattern where the received signal has acceptable level is defined by the angle 
$\phi_{3 \mathrm{~dB}}$ at which the power of the beampattern drops $3 \mathrm{~dB}$ below its maximum value and denoted as the $3 \mathrm{~dB}$ beamwidth. The characteristics of mainlobe within the $3 \mathrm{~dB}$ beamwidth are derived in [5], [19] and it is shown that the beampattern in this region is more stable and almost deterministic. To guarantee an acceptable level at the UAV, it should be flying within the $\mathrm{CB}$ mainlobe, i.e. the $3 \mathrm{~dB}$ arc-length is larger than the UAV dimensions.

For sensor nodes uniformly distributed in a cluster with radius $R$, the $3 \mathrm{~dB}$ beamwidth for the average beampattern in the $x y$-plane, i.e., $\theta=90$, is given by [5]

$$
\phi_{3 \mathrm{~dB}}=2 \sin ^{-1}\left(\frac{0.1286}{R}\right),
$$

and the corresponding $3 \mathrm{~dB}$ arc-length at distance $\rho_{a}$ is given by

$$
L_{3 \mathrm{~dB}}=\rho_{a} \phi_{3 \mathrm{~dB}}=2 \rho_{a} \sin ^{-1}\left(\frac{0.1286}{R}\right) .
$$

Therefore, the distance between the UAV and the cluster should satisfy the inequality

$$
\rho_{a} \geq \frac{L_{3 \mathrm{~dB}, \min }}{2 \sin ^{-1}\left(\frac{0.1286}{R}\right)},
$$

where $L_{3 \mathrm{~dB}, \min }$ is the minimum acceptable $3 \mathrm{~dB}$ arc-length.

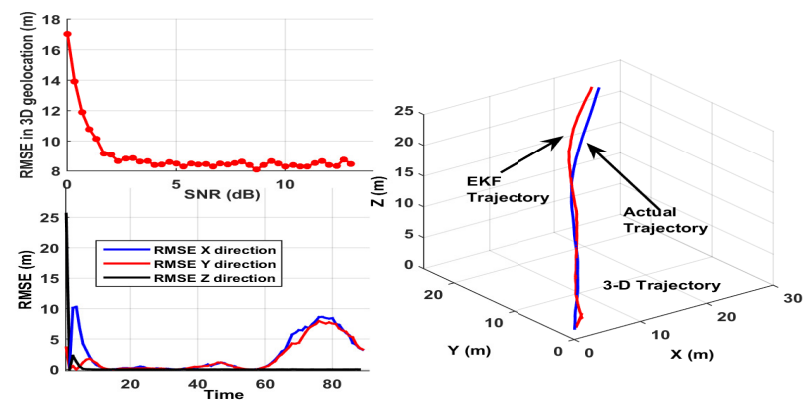

Fig. 2. RMSE performance for ADS-B based multilateration of UAV.

\section{Simulation Results}

In this section, we provide the simulation results for the proposed WSN-UAV surveillance system. The sensor nodes are randomly deployed to cover a square area of interest with dimensions $10 \mathrm{~km} \times 10 \mathrm{~km}$. Different sensor node densities $0.01,0.1$, and 1 sensor node $/ \mathrm{m}^{2}$ are assumed. The Rician $K$ factor of the channel is set to $15 \mathrm{~dB}$. The ADS-B receivers are located away from the sensing field in a safe location with minimum separation of $18.5 \mathrm{~km}$ between any two receivers. For the ADS-B system, we assume time-synchronization between different ADS-B receivers facilitated by rubidium clock standard which provides a timing synchronization accuracy of less than $20 \mathrm{~ns}$. This corresponds to a UAV geolocation error of $6 \mathrm{~m}$. Moreover, we set the maximum allowed error resulting from the TDOA localization to be $30 \mathrm{~m}$. The UAV is flying with speed of $10 \mathrm{~m} / \mathrm{s}$ thus we need to add $10 \mathrm{~m}$ to the $3 \mathrm{~dB}$ arc-length to allow $1 \mathrm{sec}$ of transmission before the the mainlobe has to be steered to the new UAV direction. In the following, we assume the total $3 \mathrm{~dB}$ arc-length to be $L_{3 \mathrm{~dB}}=50 \mathrm{~m}$ to accommodate all the aforementioned geolocation errors. We assume that the UAV is flying at $2 \mathrm{~km}$ attitude, then the minimum possible distance between the sensor nodes and the UAV is $2 \mathrm{~km}$ and it occurs when the
UAV flies directly over the sensor nodes. The CB transmission frequency is set to $915 \mathrm{MHz}$. The aforementioned settings are assumed for all the following simulations unless otherwise stated. Also, all simulation results are averaged over 1000 independent Monte Carlo runs with the sensor node locations are independently generated for each run according to uniform distribution.

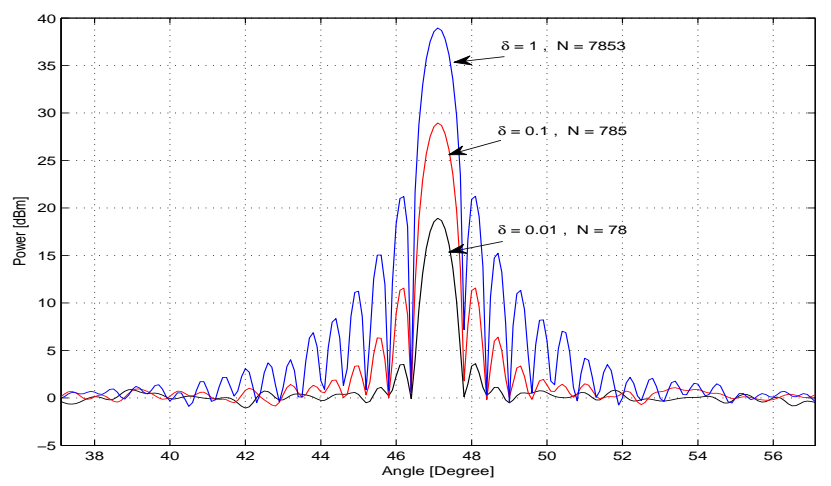

Fig. 3. Average beampattern for $\mathrm{CB}$ transmissions: $R=50 \mathrm{~m}, \rho_{a}=$ $9.7 \mathrm{~km}$, and $L_{3 \mathrm{~dB}}=50 \mathrm{~m}$.

The TDOA localization is implemented at the CU. Fig. 2 represents the TDOA geolocation estimation for over 500 runs for each SNR value. We can achieve a RMSE geolocation accuracy of $8.5 \mathrm{~m}$ at $\mathrm{SNR} \geq 3 \mathrm{~dB}$. This shows that the proposed TDOA geolocation scheme based on ADS-B can achieve almost the same geolocation accuracy compared to direct demodulation of the ADS-B frame. However, using TDOA localization enhances the security of the overall system and makes it capable to work even with GPS jamming without significantly affecting the accuracy of the overall geolocation estimate.

Now we show the performance of the CB based on the UAV location and tracking information. Fig. 3 shows the average beampattern for different node densities where we consider the array gain and ignore the FSPL. The power allocation is set to $\sqrt{P_{i}}=\sqrt{1 / N}$ and thus the total transmit power is $0 \mathrm{dBm}$. The cluster radius is kept fixed to $R=50 \mathrm{~m}$ and thus the corresponding distance between the collaborative cluster and the UAV is $\rho_{a}=9.7 \mathrm{~km}$. The total number of sensor nodes in each cluster varies for different sensor node densities. Simulations for $N=78,785$, and 7853 were carried out. Fig. 3 shows that the sensor nodes in the different clusters are able to achieve a mainlobe at the UAV direction using the CB. The mainlobe behaviour of the CB beampattern is essentially deterministic and thus the average beampattern characteristics are suitable for describing the mainlobe of a sample beampattern [20]. The array gain for the average mainlobe is higher for larger node density (i.e., larger number of sensor nodes $N$ in the cluster). The average beampattern in the sidelobe region is $0 \mathrm{dBm}$ and the mainlobe power at the UAV direction is $10 \log _{10}(1 / N)+10 \log _{10}\left(N^{2}\right)=18.9$, 28.9 , and $38.9 \mathrm{dBm}$, which matches the values obtained in Fig. 2, respectively. Alternatively, Fig. 4 shows the average beampattern for fixed number of sensor nodes $N=50$ and different node densities. In this case, the cluster radii required to obtain a cluster of $N=50$ sensor nodes are $40 \mathrm{~m}, 12.6 \mathrm{~m}$, and $4 \mathrm{~m}$, respectively. The average beampattern in the mainlobe direction is the same for the three cases $10 \log _{10}(1 / N)=17 \mathrm{dBm}$. However, the $3 \mathrm{~dB}$ beamwidth $\phi_{3 \mathrm{~dB}}$ increases for smaller cluster radius as shown in the figure and thus the distance $\rho_{a}$ is found to be $7.75,2.45$, and 
$0.77 \mathrm{~km}$, respectively. Fig. 5 shows the relation between th cluster radius $R$ and the distance to the UAV $\rho_{a}$ for differen $3 \mathrm{~dB}$ arc-length $L_{3 \mathrm{~dB}}$. The radius controls the $3 \mathrm{~dB}$ beamwidt] according to (21) and the number of sensor nodes in th cluster through the cluster area. Specifically, increasing th distance to the UAV results in more sensor nodes, hence lowe sidelobes, and smaller $3 \mathrm{~dB}$ beamwidth. Now we consider th effect of the FSPL on the received power. Using larger cluste radius $R$ increases the number of sensor nodes in each cluste and hence the array gain. However, from Fig. 5, we see tha the distance between the UAV and the cluster $\rho_{a}$ increases fo larger $R$ and consequently the FSPL increases. Fig. 6 show the effective path loss (difference between the array gain and the FSPL (in $\mathrm{dB}$ )) versus the distance $\rho_{a}$ for different sensor node densities which is linear in log scale. The efficiency of the CB increase with increasing the distance $\rho_{a}$ and it is better to have longer links between the sensor nodes and the UAV. This is opposite to the case when only one sensor node is transmitting to the UAV with no CB. There no array gain in the case to compensate for the FSPL which increases with distance.

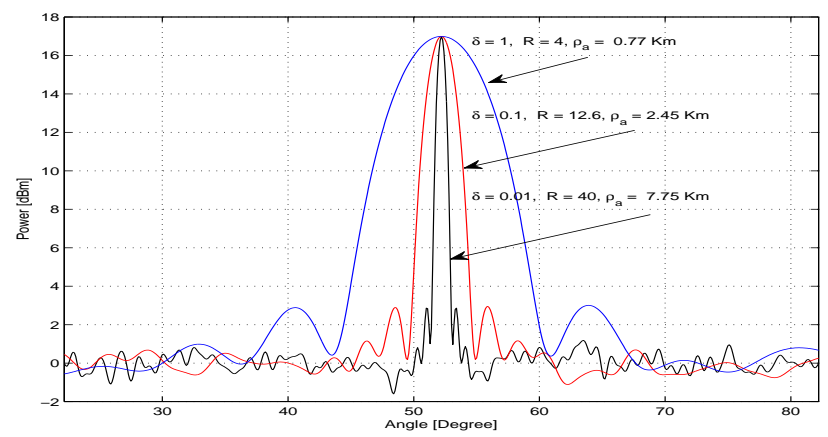

Fig. 4. Average beampattern for $\mathrm{CB}$ transmissions: $N=50$ and $L_{3 \mathrm{~dB}}=$ $50 \mathrm{~m}$.

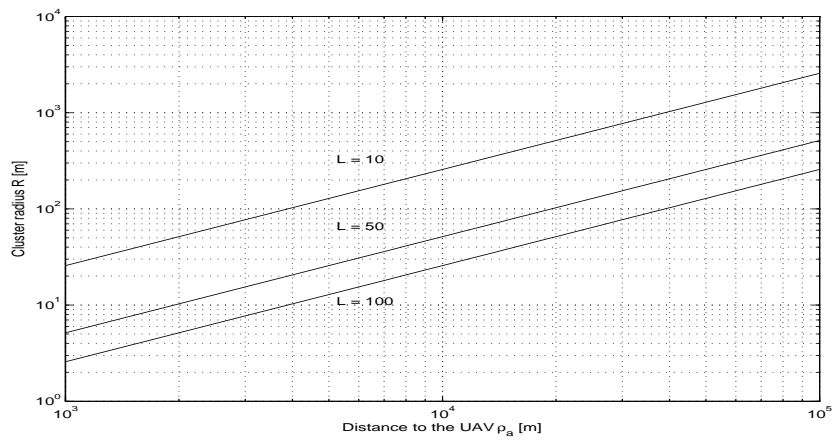

Fig. 5. Cluster radius $R$ vs. distance $\rho_{a}$ between the UAV and the cluster.

\section{CONCLUSION}

This paper proposed WSN-UAV surveillance system for military remote monitoring and surveillance. We have showed the feasibility of the location and tracking information of the UAV obtained from TDOA multilatration based on the ADSB signals for the CB. Simulation results have shown that the proposed system can accommodate the geolocalization errors and UAV movement. CB is more suitable for long distance transmissions than direct transmission.

\section{REFERENCES}

[1] P. Mario, F. Fontan, M. Dominguez, and S. Otero, "Bio-surveillance monitoring with a wireless network," in 19th International Conference on Systems Engineering, 2008. ICSENG '08., Aug. 2008, pp. 389-394.

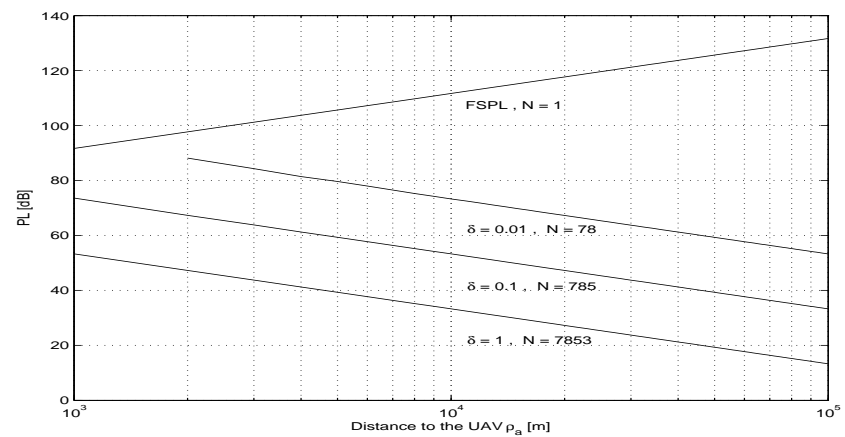

Fig. 6. Effective path loss PL (i.e. the difference between the FSPL and the array gain) vs. distance $\rho_{a}$ between the UAV and the cluster.

[2] A. Mainwaring, D. Culler, J. Polastre, R. Szewczyk, and J. Anderson, "Wireless sensor networks for habitat monitoring," in Proc. 1st ACM International Workshop on Wireless Sensor Networks and Applications, WSNA'02, 2002, pp. 88-97.

[3] D. Mascarenas, E. Flynn, C. Farrar, G. Park, and M. Todd, "A mobile host approach for wireless powering and interrogation of structural health monitoring sensor networks," IEEE Sensors Journal, vol. 9, no. 12 , pp. 1719-1726, Dec. 2009.

[4] A. Goncalves-Coelho, L. Veloso, and V. Lobo, "Tests of a light UAV for naval surveillance," in OCEANS 2007 - Europe, Jun. 2007, pp. 1-4

[5] H. Ochiai, P. Mitran, H. V. Poor, and V. Tarokh, "Collaborative beamforming for distributed wireless ad hoc sensor networks," IEEE Transactions on Signal Processing, vol. 53, no. 11, pp. 4110-4124, Nov. 2005.

[6] M. F. A. Ähmed and S. A. Vorobyov, "Collaborative beamforming for wireless sensor networks with gaussian distributed sensor nodes," IEEE Transactions on Wireless Communications, vol. 8, no. 2, pp. 638-643, Feb. 2009.

[7] D. Torrieri, "Statistical theory of passive location systems," Aerospace and Electronic Systems, IEEE Transactions on, vol. AES-20, no. 2, pp. 183-198, March 1984.

[8] Y. Chan and K. Ho, "A simple and efficient estimator for hyperbolic location," Signal Processing, IEEE Transactions on, vol. 42, no. 8, pp. 1905-1915, Aug 1994

[9] L. Villas, D. Guidoni, and J. Ueyama, "3D localization in wireless sensor networks using unmanned aerial vehicle," in Network Computing and Applications (NCA), 2013 12th IEEE International Symposium on, Aug 2013, pp. 135-142.

[10] W. Neven, T. Quilter, R. Weedon, and R. Hogendoorn, Report on EATMP TRS 131/04, Wide Area Multilateration. Nationaal LuchtNationaal Lucht- en Ruimtevaartlaboratorium en Ruimtevaartlaboratorium en Ruimtevaartlaboratorium, 2005.

[11] M. Ahmed and S. Vorobyov, "Power control for collaborative beamforming in wireless sensor networks," in Signals, Systems and Computers (ASILOMAR), 2011 Conference Record of the Forty Fifth Asilomar Conference on, Nov 2011, pp. 99-103.

[12] D. R. Brown and H. V. Poor, "Time-slotted round-trip carrier synchronization for distributed beamforming," IEEE Transactions on Signal Processing, vol. 56, no. 11, pp. 5630-5643, Nov. 2008.

[13] M. Ahmed and S. A. Vorobyov, "Sidelobe control in collaborative beamforming via node selection," IEEE Transactions on Signal Processing, vol. 58, no. 12, pp. 6168-6180, Dec. 2010.

[14] A. P. Petropulu, L. Dong, and H. V. Poor, "Weighted cross-layer cooperative beamforming for wireless networks," IEEE Transactions on Signal Processing, vol. 57, no. 8, pp. 3240-3252, Aug. 2009.

[15] E. Haas, "Aeronautical channel modeling," IEEE Transactions on Vehicular Technology, vol. 51, no. 2, Mar. 2002.

[16] Symmetricom, Rubidium Frequency Standard 8040C. San Jose: Microsemi, 2011.

[17] H.-J. Du and P. Y. Lee, "Simulation of multi-platform geolocation using a hybrid TDOA-AOA method," Technical Memorandum Defense $R$ and D Canada, Dec. 2004.

[18] A. Shareef and Y. Zhu, "Localization using Extended Kalman Filters in Wireless Sensor Networks," Kalman Filter: Recent Advances and Applications, Edited by V. M. Moreno and A. Pigazo, Chapter 13, Apr. 2009, InTech, Vienna, Austria.

[19] M. F. A. Ahmed and S. A. Vorobyov, "Beampattern random behavior in wireless sensor networks with gaussian distributed sensor nodes," in Proc. Canadian Conference on Electrical and Computer Engineering, CCECE'08, May 2008, pp. 257-260.

[20] M. Ahmed and S. Vorobyov, "Beampattern random behavior in wireless sensor networks with gaussian distributed sensor nodes," in Electrical and Computer Engineering, 2008. CCECE 2008. Canadian Conference on, May 2008, pp. 257-260. 